\title{
Annexin A5-Conjugated Polymeric Micelles for Dual SPECT and Optical Detection of Apoptosis
}

\author{
Rui Zhang ${ }^{1}$, Wei $\mathrm{Lu}^{1}$, Xiaoxia Wen ${ }^{1}$, Miao Huang ${ }^{1}$, Min Zhou ${ }^{1}$, Dong Liang ${ }^{2}$, and Chun $\mathrm{Li}^{1}$ \\ ${ }^{1}$ Department of Experimental Diagnostic Imaging, University of Texas M.D. Anderson Cancer Center, Houston, Texas; and ${ }^{2}$ College \\ of Pharmacy, Texas Southern University, Houston, Texas
}

\begin{abstract}
Imaging of apoptosis can allow noninvasive assessment of disease states and response to therapeutic intervention for a variety of diseases. The purpose of this study was to develop and evaluate a multimodal nanoplatform for the detection of apoptosis. Methods: To modulate the pharmacokinetics of annexin A5, a 36-kDa protein that binds specifically with phosphatidylserine, annexin A5 was conjugated to polyethylene glycol-coated, core-cross-linked polymeric micelles (CCPMs)
\end{abstract} dually labeled with near-infrared fluorescence fluorophores and a radioisotope $\left({ }^{111} \mathrm{In}\right)$. To evaluate the specificity of the binding of annexin A5-CCPM to apoptotic cells, both fluorescence microscopy and cell-binding studies were performed in vitro. Pharmacokinetics, biodistribution, dual nuclear and optical imaging, and immunohistochemical studies were performed in 2 xenografted tumor models to evaluate the potential applications of annexin A5-CCPM. Results: In cell-based studies, annexin A5-CCPM exhibited strongly specific binding to apoptotic tumor cells. This binding could be efficiently blocked by annexin A5. In mice, annexin A5-CCPM displayed a mean elimination half-life of $12.5 \mathrm{~h}$. The mean initial concentration in blood was $22.4 \%$ of the injected dose/mL, and annexin A5-CCPM was mainly distributed in the central blood compartment. In mice bearing EL4 lymphoma treated with cyclophosphamide and etoposide and in mice bearing MDA-MB-468 breast tumors treated with poly(L-glutamic acid)-paclitaxel and cetuximab (IMC-C225) anti-epidermal growth factor receptor antibody, the tumor apoptosis was clearly visualized by both SPECT and fluorescence molecular tomography. In contrast, there was little accumulation of this nanoradiotracer in the tumors of untreated mice. The biodistribution data were consistent with the imaging data, with tumor-to-muscle and tumor-to-blood ratios of 38.8 and 4.1 , respectively, in treated mice, and 14.8 and 2.2, respectively, in untreated mice bearing EL4 lymphoma. Moreover, further studies demonstrated that the conventional $99 \mathrm{~m}$ Tc-labeled hydrazinonicotinamide annexin A5 and the plain CCPM control exhibited significantly lower uptake in the tumors of the treated mice than annexin A5-CCPM. Immunohistochemistry staining study showed that radioactivity count correlated with fluorescence signal from the nanoparticles, and both signals colocalized with the region of tumor apoptosis. Conclusion: Annexin A5-CCPM allowed visualization of tumor apoptosis by both nuclear and optical techniques. The comple-

Received Sep. 23, 2010; revision accepted Jan. 31, 2011.

For correspondence or reprints contact: Chun Li, Department of Experimental Diagnostic Imaging-Unit 59, University of Texas M.D. Anderson Cancer Center, 1515 Holcombe Blvd., Houston, TX 77030.

E-mail: cli@mdanderson.org

COPYRIGHT @ 2011 by the Society of Nuclear Medicine, Inc.

mentary information acquired with multiple imaging techniques should be advantageous in assessing and validating early response to therapy.

Key Words: apoptosis; annexin A5; polymeric micelles; nuclear imaging; fluorescence optical imaging

J Nucl Med 2011; 52:958-964

DOI: $10.2967 /$ jnumed.110.083220

A to homeostasis, normal development, and physiology. Dysregulation of apoptosis can lead to the accumulation of unwanted cells, such as occurs in cancer, and the removal of needed cells or disorders of normal tissues, such as heart, neurodegenerative, and autoimmune diseases (1-4). Because apoptosis is associated with many diseases, noninvasive detection of apoptosis has received much attention. To date, several biochemical features in cells have been identified and used for the visualization of apoptosis. Phosphatidylserine, a component of cell membrane phospholipids in the inner plasma membrane, redistributes and externalizes to the outer surface of the membrane in the early stage of apoptosis $(5,6)$. For the detection of phosphatidylserine on the surface of cells, annexin A5 can be used as a targeting ligand. Annexin A5 (molecular weight, $36 \mathrm{kDa}$ ) is an endogenous human protein that consists of 319 amino acids. Annexin A5 binds strongly and specifically with phosphatidylserine residues (6-11). In past decades, various annexin A5 derivatives have been developed for the detection of apoptosis with different imaging modalities, including fluorescence (12,13), PET (14-17), SPECT (18-25), and MRI $(26,27)$. A few of these agents have shown promising results in imaging apoptosis in preclinical studies and clinical trials $(18-21,23,25)$.

The timing of annexin A5 imaging after therapy has been shown to be critical (28). If peak apoptotic activity after administration of anticancer therapy varies from patient to patient and from treatment to treatment, then it will be necessary to determine for each patient the best time to scan after the start of chemotherapy. Because annexin A5 has a short blood half-life of only a few minutes, it has been suggested that an imaging protocol using multiple separate 
injections of ${ }^{99 \mathrm{~m} T c-l a b e l e d ~ a n n e x i n ~ A 5 ~ a n d ~ m u l t i p l e ~ r a d i o-~}$ nuclide scans could be used to assess peak apoptotic activity (28). Such a protocol, however, might be difficult to implement in larger-scale trials. One approach to address this limitation is to modulate the pharmacokinetics of annexin A5 to ensure that the protein has sufficient time to reach its target over the course of action of apoptosisinducing agents. In this work, we developed and evaluated micelle-modified annexin A5 with prolonged blood circulation time. We hypothesized that an imaging probe based on annexin A5 with a long blood half-life would make it possible to capture therapy-induced apoptotic cells over a prolonged course rather than a snapshot of the drug's action. The micelles were also dually labeled with a $\gamma$-emitter, ${ }^{111} \mathrm{In}$, and a near-infrared fluorescent indocyanine 7 (Cy7)-like dye to permit simultaneous visualization of apoptosis by both nuclear and optical imaging.

\section{MATERIALS AND METHODS}

\section{Synthesis and Characterization of Annexin A5-Coated, Core-Cross-Linked Polymeric Micelles (CCPMs)}

The synthesis, characterization, and radiolabeling procedures for ${ }^{111}$ In-labeled annexin A5-CCPM are described in the supplemental materials (available online only at http://jnm.snmjournals. org) (29).

\section{Fluorescence Microscopy}

DLD-1 human colorectal adenocarcinoma cells were obtained from American Type Culture Collection. Cells were grown in Dulbecco's modified Eagle's medium and a nutrient mixture of F12 Ham (DMEM/F12) (Gibco) supplemented with 10\% fetal calf serum and a mixture of antibiotics (penicillin [100 units $\mathrm{mL}^{-1}$ ] and streptomycin $\left[0.1 \mathrm{mg} \mathrm{mL}^{-1}\right]$; Biochrom AG). Cells were grown at $37^{\circ} \mathrm{C}$ in a humidified atmosphere and $5 \% \mathrm{CO}_{2}$. DLD-1 cells were treated with tumor necrosis factor-related apoptosisinducing ligand (TRAIL) (Sigma-Aldrich) $(200 \mu \mathrm{L}, 150 \mathrm{ng} / \mathrm{mL})$ for $2 \mathrm{~h}$ to induce apoptosis. The cells were then incubated with annexin A5-CCPM or CCPM in $N$-(2-hydroxyethyl)piperazine$N^{\prime}$ '-(2-ethanesulfonic acid) (HEPES) binding buffer $(25 \mathrm{mM}$ HEPES, $140 \mathrm{mM} \mathrm{NaCl}$, and $2.5 \mathrm{mM} \mathrm{CaCl}_{2}, \mathrm{pH}$ 7.4) for $15 \mathrm{~min}$ at $37^{\circ} \mathrm{C}$ at a final concentration of $20 \mathrm{nM}$ nanoparticles. Untreated DLD- 1 cells were used as a control. Cells were washed 3 times with HEPES binding buffer, and cell membrane was stained with wheat germ agglutinin-Alexa Fluor 594 (Invitrogen). The cell samples were transferred onto Lab-Tek II chambered cover glass (ThermoScientific) and visualized under an Axio Observer.Z1 fluorescence microscope (Carl Zeiss MicroImaging $\mathrm{GmbH}$ ) equipped with Cy7 filters (wavelength, 710/810 nm) and rhodamine filters (wavelength, 570/620 nm).

\section{Cell-Binding Study}

To study the cell uptake of ${ }^{111}$ In-labeled annexin A5-CCPM, DLD-1 cells were grown in 6-cm Petri dishes to subconfluent densities in DMEM/F12 containing 10\% fetal bovine serum $1 \mathrm{~d}$ before experiments. DLD-1 cells were treated with TRAIL at doses of $1.5 \mathrm{ng} / \mathrm{mL}, 15 \mathrm{ng} / \mathrm{mL}$, and $150 \mathrm{ng} / \mathrm{mL}$ for $2 \mathrm{~h}$ to induce apoptosis. After treatment with TRAIL, the medium was replaced with $2 \mathrm{~mL}$ of fresh medium containing ${ }^{111}$ In-labeled annexin
A5-CCPM nanoparticles $(\sim 1.8 \mathrm{MBq} / \mathrm{mL})$, and cells were incubated for $15 \mathrm{~min}$. The cell monolayers were scraped and transferred into 5 -mL tubes, and the tubes were briefly stirred in a vortex mixer. Aliquots of DLD-1 cell suspension $(100 \mu \mathrm{L})$ were transferred into a microcentrifuge tube containing $500 \mu \mathrm{L}$ of a $75: 25$ mixture of silicon oil (density, 1.05; Aldrich) and mineral oil (density, 0.872; Acros). The mixture was centrifuged at 14,000 rpm for $5 \mathrm{~min}$. After the tubes were frozen with liquid nitrogen, the bottom tips containing the cell pellet were cut off. The cell pellets and the supernatants were counted with a $\gamma$-counter (Perkin-Elmer). The protein content in $100 \mu \mathrm{L}$ of cell suspension was quantified in a separate experiment using a Bio-Rad protein assay kit according to the manufacturer's protocol. Activity ratios of the cell pellet to medium ([cpm/ $\mu \mathrm{g}$ of protein in pellet]/[cpm/ $\mu \mathrm{g}$ of medium]) were calculated and plotted against time. The experiments were performed in pentaplicate. In a separate study, aliquots of DLD-1 cell suspension $(500 \mu \mathrm{L})$ after TRAIL treatment were transferred into microcentrifuge tubes, annexin A5-fluorescein isothiocyanate was added to the tubes, and the percentage of apoptotic cells was analyzed using a FACSCalibur flow cytometer (BD Biosciences).

\section{Pharmacokinetic Study}

All animal studies were performed in the Small Animal Imaging Facility at the University of Texas M.D. Anderson Cancer Center in accordance with institutional guidelines. For the pharmacokinetic study, healthy female Swiss mice (22-25 g; Charles River Laboratories) $(n=8)$ were injected intravenously at a dose of $5 \times 10^{13}$ nanoparticles per mouse (1.8 MBq/mouse). At predetermined intervals, blood samples $(10 \mu \mathrm{L})$ were taken from the tail vein, and the radioactivity of each sample was measured with a Cobra Autogamma counter (Packard). The blood pharmacokinetic parameters for the radiotracer were analyzed using a noncompartmental model with WinNonlin 5.0.1 software (Pharsight Corp.).

\section{Tumor Models}

Apoptosis of EL4 lymphoma was induced as described previously (30). Briefly, EL4 cells $\left(1.0 \times 10^{6}\right)$ were inoculated subcutaneously in the right flank of 6- to 8-wk-old syngeneic C57BL/6 mice. Two weeks after inoculation, when tumors reached approximately $5-6 \mathrm{~mm}$ in diameter, mice were divided into 4 groups. Mice in groups 1, 3, and 4 were given an intraperitoneal injection of cyclophosphamide $(25 \mathrm{mg} / \mathrm{kg})$ and etoposide $(19 \mathrm{mg} / \mathrm{kg})$. Mice in group 2 were not treated and were used as a control group. Mice in groups 1 and 2 received intravenous injection of ${ }^{111}$ In-labeled annexin A5-CCPM $1 \mathrm{~d}$ after drug treatment $\left(n=7\right.$ per group). Mice in group 3 received ${ }^{99 \mathrm{~m}} \mathrm{Tc}$-hydrazinonicotinamide (HYNIC) annexin A5, and mice in group 4 received ${ }^{111}$ In-labeled CCPM $1 \mathrm{~d}$ after drug treatment $(n=5$ per group).

Induction of apoptosis in an MDA-MB-468 breast cancer model was achieved as described previously (31). Briefly, MDAMB-468 cells $\left(1 \times 10^{6}\right)$ were inoculated subcutaneously in the right flank of 6- to 8-wk-old female nude mice. Four weeks after inoculation, when tumors reached approximately $5-6 \mathrm{~mm}$ in average diameter, mice were divided into 2 groups ( $n=2$ per group). Mice in group 1 were given a single intravenous injection of poly (L-glutamic acid)-paclitaxel at a dose of $100 \mathrm{mg}$ equivalent of paclitaxel per kilogram on day 1 and a single intraperitoneal injection of cetuximab (Imclone Systems), a monoclonal antibody directed against epidermal growth factor receptor, at a dose of 1 
$\mathrm{mg}$ on day 4. Mice in group 2 were not treated and were used as a control group. ${ }^{111}$ In-labeled annexin A5-CCPM was injected via the tail vein $1 \mathrm{~d}$ after the injection of cetuximab.

\section{Imaging and Biodistribution Studies}

${ }^{111}$ In-labeled annexin A5-CCPM $\left(1 \times 10^{14}\right.$ particles per mouse, 9.2 $\mathrm{MBq} /$ mouse) was injected via the tail vein into tumor-bearing mice after chemotherapy. At $48 \mathrm{~h}$ after administration of ${ }^{111} \mathrm{In}-$ labeled annexin A5-CCPM, SPECT and CT images were acquired using an xSPECT-CT scanner (Gamma Medica). SPECT scans (3$\mathrm{cm}$ radius of rotation, 32 projections, $20 \mathrm{~s} /$ projection) and CT scans (512 projections, $75 \mathrm{kV}, 500 \mathrm{~mA}$ ) were acquired and coregistered for image fusion and presentation of 3-dimensional anatomic localization of the tracer signal. Acquired SPECT and CT datasets were processed with AMIRA software (version 5.1). The whole-body optical imaging was performed using a fluorescent molecular tomograph (Visen) equipped with an excitation-emission wavelength filter (760-790 nm). During each imaging session, mice were anesthetized with $2 \%$ isoflurane gas (Iso-Thesia) in oxygen.

Mice in group 3 injected with ${ }^{99 \mathrm{~m} T c-H Y N I C}$ annexin A5 were killed at $6 \mathrm{~h}$ after radiotracer injection. Mice in the other 3 groups were killed at $48 \mathrm{~h}$ after administration of either ${ }^{111} \mathrm{In}$-labeled annexin A5-CCPM or ${ }^{111}$ In-labeled CCPM. Various tissues were removed, weighed, and counted for radioactivity with a Cobra Autogamma counter (Packard). Uptake of the nanoparticles was calculated as the percentage of the injected dose per gram of tissue (\% ID/g). In addition, fluorescence images of dissected tissues were acquired using an IVIS imaging system (Xenogen Corp.) equipped with indocyanine green filter sets (excitation/emission, 710-760/810-875 nm). Fluorescence intensities were calculated using software (Living Image, version 2.11; Xenogen). Uptake of the nanoparticles in various tissues was calculated as flux (photons/s) per gram of tissue. A Student $t$ test was used to compare differences in tissue uptake between different groups, and $P$ values less than 0.01 were considered highly significant.

\section{Autoradiography and Optical Imaging}

Tumors harvested at the end of the imaging sessions were snapfrozen and sectioned into 5 - $\mu \mathrm{m}$ sections. The sections were photographed and exposed on BAS-SR 2025 Fuji phosphorous films. The film was scanned with a FLA5100 Multifunctional Imaging System (Fujifilm Medical Systems USA).

After autoradiographic study, optical images of the sections were acquired by scanning at $800 \mathrm{~nm}$ using an Odyssey infrared imaging system (LI-COR Biosciences).

\section{Immunohistochemistry}

After autoradiographic study, 1 slice of each tumor was immunostained with anticaspase- 3 antibody using a commercial kit (Sigma-Aldrich) according to the manufacturer's protocol. The stained sections were counterstained with hematoxylin. Images were recorded using a Zeiss Axio Observer.Z1 microscope.

For terminal deoxynucleotidyl transferase dUTP nick end labeling (TUNEL), a slide adjacent to those used for autoradiographic studies was stained with a TUNEL staining kit (R\&D Systems) according to the manufacturer's instructions. Cell nuclei were counterstained with 4'-6-diamidino-2-phenylindole. The cellular fluorescence was examined under a Zeiss Axio Observer.Z1 fluorescence microscope equipped with ultraviolet filter for 4'-6diamidino-2-phenylindole, 494/517-nm filter for TUNEL staining, and 710/810-nm filter for Cy7.

\section{Statistical Analysis}

Statistical analysis was performed with GraphPad Prism software (version 4.0). Unless otherwise stated, group comparisons were made using standard ANOVA methods. Groups with a $P$ value less than 0.01 were considered highly significant.

\section{RESULTS}

\section{Characterization of Annexin A5-CCPM}

The reaction scheme for the conjugation of annexin A5 to the surface of CCPM is shown in Supplemental Figure 1A. Supplemental Figure $1 \mathrm{~B}$ is a transmission electron microscopic image of annexin A5-CCPM; these images showed that the average diameter of the nanoparticles was $25 \mathrm{~nm}$. Each annexin A5-CCPM nanoparticle contained approximately 40 annexin A5 molecules on the surface, calculated on the basis of the molar feed ratio. The excitation and emission light intensities for the annexin A5-CCPM nanoparticles peaked at $755 \mathrm{~nm}$ and $781 \mathrm{~nm}$, respectively (Supplemental Fig. 1C). As indicated by instant thin-layer chromatography, the labeling efficiency of annexin A5-CCPM was greater than 98\% without further purification. The specific activity was $185 \mathrm{MBq} / \mathrm{nmol}$ of nanoparticles.

\section{In Vitro Binding to Apoptotic Cells}

Supplemental Figure 2A shows fluorescence microscopic images of viable human DLD-1 colon cancer cells and apoptotic DLD-1 cells incubated with annexin A5-CCPM. The membrane of cells treated with both TRAIL and phosphate-buffered saline was stained red with wheat germ agglutinin-Fluor594. However, only the TRAIL-treated cells showed binding of annexin A5-CCPM to the cell membrane. Moreover, the binding of annexin A5-CCPM to the apoptotic cells was efficiently blocked by annexin A5 (Supplemental Fig. 2A).

TRAIL-induced apoptosis in DLD-1 cells was analyzed by flow cytometry at TRAIL concentrations of 1.5-150 ng/ $\mathrm{mL}$ using annexin A5-fluorescein isothiocyanate (Supplemental Fig. 2B). There was a sharp increase in the apoptotic response when TRAIL concentration increased from 15 to $150 \mathrm{ng} / \mathrm{mL}$ and the percentage of apoptotic cells increased from $3.76 \%$ to $24.5 \%$. When ${ }^{111}$ In-labeled annexin A5CCPM was incubated with TRAIL-treated DLD-1 cells, an increasing amount of the radiotracer was bound to the cells in a TRAIL dose-dependent manner (Supplemental Fig. 2C). A significant increase in cell-associated radioactivity was detected at a TRAIL dose of $1.5 \mathrm{ng} / \mathrm{mL}$. The binding of ${ }^{111} \mathrm{In}$-labeled annexin A5-CCPM to apoptotic cells was completely blocked by an excess of annexin A5 (Supplemental Fig. 2C). These results confirmed that ${ }^{111} \mathrm{In}$ labeled annexin A5-CCPM selectively bound to phosphatidylserine on the surface of apoptotic cells.

\section{Pharmacokinetics}

Supplemental Figure 3 compares the activity-time profiles of ${ }^{111}$ In-labeled annexin A5-CCPM and plain CCPM. Data for CCPM were taken from the study by Yang et al. 
(29). The plain CCPM showed a biexponential disposition, whereas ${ }^{111}$ In-labeled annexin A5-CCPM appeared to have a single exponential disposition after intravenous administration. Mean pharmacokinetic parameters are summarized in Table 1. Compared with unmodified CCPM, the introduction of annexin A5 to CCPM resulted in a significantly shorter mean terminal elimination half-life, less systemic exposure (total area-under-the-blood concentration versus time curve), and smaller mean volume of distribution at steady state. The mean systemic clearance was significantly slower with the unmodified CCPM $(0.0902 \mathrm{~mL} / \mathrm{h})$ than with ${ }^{111}$ In-labeled annexin A5-CCPM $(0.217 \mathrm{~mL} / \mathrm{h}, P<$ 0.001 ), suggesting that ${ }^{111}$ In-labeled annexin A5-CCPM was cleared more than twice as quickly as the unmodified CCPM. This faster clearance can be attributed to higher elimination by the liver or spleen of ${ }^{111}$ In-labeled annexin A5-CCPM than of CCPM. For both ${ }^{111}$ In-labeled CCPM and annexin A5-CCPM, mean volume of distribution at steady state was similar to mean volume of distribution in the central compartment, indicating that both agents mainly distributed to the central compartment (systemic blood circulation).

\section{Biodistribution}

Treatment of mice bearing EL4 lymphoma with cyclophosphamide and etoposide induces substantial apoptosis (30). This model was used to study the effect of apoptosis on the biodistribution of ${ }^{111}$ In-labeled annexin A5-CCPM (Fig. 1). At $48 \mathrm{~h}$ after injection, ${ }^{111}$ In-labeled annexin A5CCPM showed significantly higher uptake in the tumors of the treated mice $(8.01 \% \mathrm{ID} / \mathrm{g})$ than in the tumors of the untreated mice $(3.2 \% \mathrm{ID} / \mathrm{g})(P<0.001)$ (Fig. 1A). The tumor-to-blood ratios were 2.2 in the untreated group versus 4.1 in the cyclophosphamide- and etoposide-treated groups, and the tumor-to-muscle ratios were 14.8 in the untreated group versus 38.8 in the chemotherapy-treated group. The biodistribution was also determined by analysis of fluorescence signal intensities of the resected tissues (Supplemental Fig. 4). These data were consistent with the data obtained by the radioactivity count method. Spleens of the mice treated with chemotherapy showed significantly higher uptake of ${ }^{111}$ In-labeled annexin A5CCPM than did spleens in the control group $(P<0.001)$, probably because of cyclophosphamide-induced apoptosis of this tissue (20). ${ }^{111}$ In-labeled annexin A5-CCPM (8.01\% ID/g) also showed significantly higher uptake in the tumors of the treated mice than ${ }^{99 \mathrm{~m}} \mathrm{Tc}-\mathrm{HYNIC}$ annexin A5 $(4.14 \%$ ID/g) and ${ }^{111} \mathrm{In}-$ labeled CCPM $(2.81 \% \mathrm{ID} / \mathrm{g})(P<0.001)$ (Fig. 1B).

\section{Nuclear and Optical Imaging}

Figures 2 and 3 compare micro-SPECT and fluorescent molecular tomography optical images of mice without treatment and mice treated with a regimen of cyclophosphamide-etoposide. The images were obtained $48 \mathrm{~h}$ after administration of ${ }^{111}$ In-labeled annexin A5-CCPM. The nuclear images were generally in concordance with the biodistribution data: there was relatively high accumulation in the liver and spleen. The apoptotic tumor was clearly visualized after chemotherapy in these mice with EL4 lymphoma (Fig. 3A). In contrast, there was little signal in the tumors of untreated mice (Fig. 2A). In optical images, tumors were readily visualized in mice after chemotherapy, whereas tumors in the untreated mice were not detected (Figs. 3B and 2B, respectively). Similar findings were observed in mice bearing subcutaneous MDA-MB-468 breast cancer (Supplemental Fig. 5).

\section{Autoradiography and Immunohistochemistry}

Intratumoral distribution of ${ }^{111} \mathrm{In}-$ labeled annexin A5CCPM was shown in both autoradiographic images (Figs. 2C and 3C and Supplemental Fig. 5C) and fluorescence optical images of tumor sections (Figs. 2D and 3D and Supplemental Fig. 5D). Chemotherapy caused markedly increased radioactivity and fluorescent signal intensity in both tumor models. In the EL4 lymphoma model, localization of both radioactivity and fluorescence signal from ${ }^{111}$ In-labeled annexin A5-CCPM correlated with apoptotic

TABLE 1

Comparison of Pharmacokinetic Parameters (Mean \pm SD) for Plain CCPM and ${ }^{111}$ In-Labeled Annexin A5-CCPM in Mice

\begin{tabular}{|c|c|c|c|}
\hline Parameter & CCPM & Annexin A5-CCPM & $P$ \\
\hline No. of mice & 8 & 7 & - \\
\hline Terminal biologic half-life (h) & $39.0 \pm 8.4$ & $12.5 \pm 1.4$ & 0.000 \\
\hline AUC (\%ID/mL blood) & $1148 \pm 213$ & $466 \pm 53$ & 0.000 \\
\hline $\mathrm{C}_{\max }(\% \mathrm{ID} / \mathrm{mL})$ & $27.2 \pm 3.1$ & $22.4 \pm 2.9$ & 0.008 \\
\hline $\mathrm{V}_{\mathrm{d}}(\mathrm{mL})$ & $4.95 \pm 0.9$ & $3.91 \pm 0.64$ & 0.024 \\
\hline $\mathrm{V}_{\mathrm{ss}}(\mathrm{mL})$ & $5.18 \pm 0.65$ & $4.44 \pm 0.24$ & 0.015 \\
\hline $\mathrm{CL}(\mathrm{mL} / \mathrm{h})$ & $0.0902 \pm 0.019$ & $0.217 \pm 0.023$ & 0.000 \\
\hline MRT (h) & $58.7 \pm 8.3$ & $20.6 \pm 1.9$ & 0.000 \\
\hline
\end{tabular}

$\mathrm{AUC}=$ total area under the blood concentration versus time curve; $\mathrm{C}_{\max }=$ predicted maximum drug concentration in blood; $\%$ ID $=$ percentage of injected dose; $\mathrm{V}_{\mathrm{d}}=$ apparent volume of distribution; $\mathrm{V}_{\mathrm{ss}}=$ steady-state volume of distribution; $\mathrm{CL}=$ total body clearance; $\mathrm{MRT}=$ mean residence time.

$P$ values were obtained using 2 -sample $t$ test. 


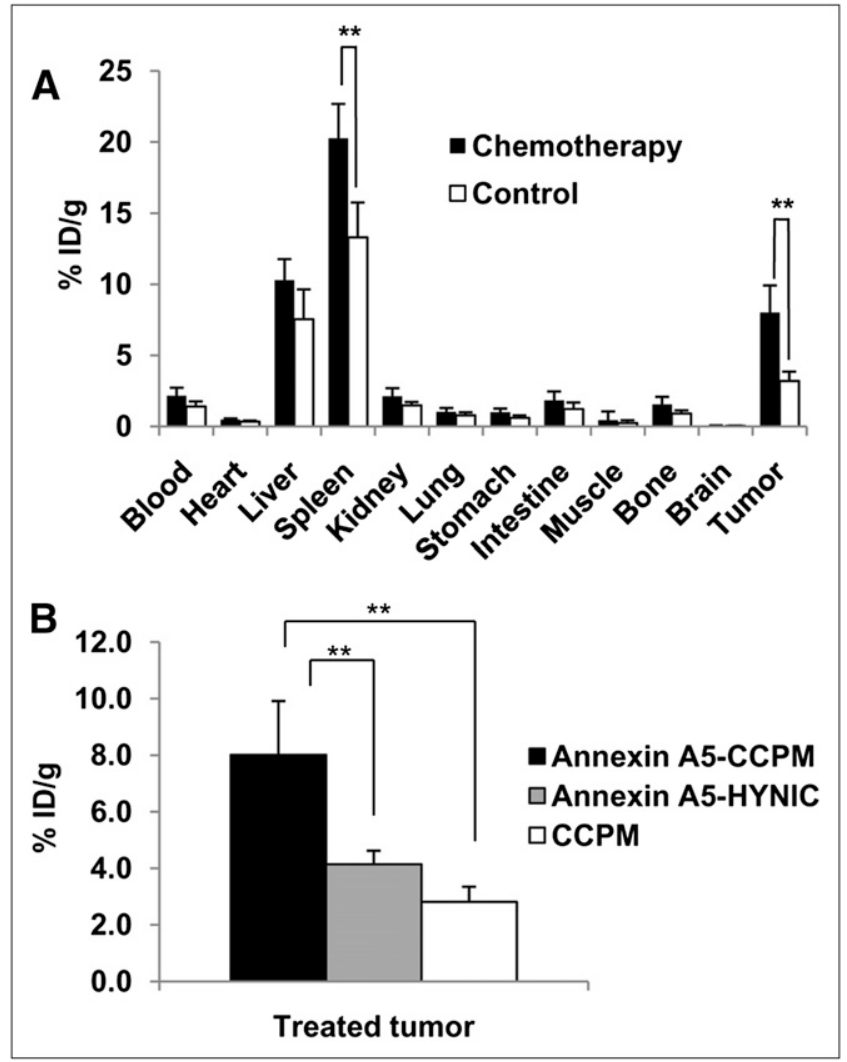

FIGURE 1. Biodistribution in mice bearing EL4 lymphoma. (A) Biodistribution $48 \mathrm{~h}$ after administration of ${ }^{111}$ In-labeled annexin A5CCPM. Mice in treatment group were injected with ${ }^{111} \mathrm{In}$-labeled annexin A5-CCPM intravenously $24 \mathrm{~h}$ after administration of cyclophosphamide and etoposide. Mice in control group were injected only with ${ }^{111} \mathrm{In}$-labeled annexin A5-CCPM. (B) Tumor uptake of ${ }^{111}$ In-labeled annexin A5-CCPM, ${ }^{111}$ In-labeled CCPM, and ${ }^{99 m T C-}$ HYNIC annexin A5 in EL4 tumor of mice treated with cyclophosphamide and etoposide. Data obtained using radioactivity count method are plotted as \%ID/g. All data are expressed as mean \pm SD. ${ }^{\star \star} P=0.001$

cells stained with caspase- 3 antibody (Figs. $2 \mathrm{C}-2 \mathrm{~F}$ and $3 \mathrm{C}-$ 3F). In both the EL4 lymphoma model and the MDA-MB468 models, the fluorescent signal from ${ }^{111}$ In-labeled annexin A5-CCPM colocalized with apoptotic cells detected with TUNEL assay (Fig. 4 and Supplemental Fig. 6).

\section{DISCUSSION}

In this study, we found that injection of ${ }^{111}$ In-labeled annexin A5-CCPM allowed ready visualization of chemotherapy-induced apoptosis with both SPECT and near-infrared fluorescence imaging in 2 separate xenograft models in mice. Moreover, ${ }^{111}$ In-labeled annexin A5-CCPM permitted detection of apoptotic cells at the microscopic level by optical imaging.

Apoptosis is a dynamic process in which newly generated apoptotic cells are rapidly removed by phagocytic macrophages (32). Therefore, there is a short time window for detection of apoptotic cells. Because of its short blood half-life $(<7 \mathrm{~min})(33),{ }^{99 \mathrm{~m}} \mathrm{Tc}-$ labeled annexin A5 exhibits limited exposure time and penetration into tumors, which compromise its sensitivity. We hypothesized that modulation of the pharmacokinetics of annexin A5 through the use of long-circulating nanoparticles would permit annexin A5 nanoparticles to penetrate deep into the tumor mass and to visualize apoptotic cells over a prolonged period, allowing improved detection of therapy-induced apoptosis. In this study, annexin A5 was conjugated to the surface of polyethylene glycol-coated CCPM. Although introduction of annexin A5 molecules to CCPM resulted in a significant reduction in the blood half-life of the resulting annexin A5-CCPM, as compared with the unmodified CCPM (Table 1), the mean half-life of $12.5 \mathrm{~h}$ was still much longer than that of annexin A5. We found that although conventional ${ }^{99 \mathrm{~m} T c-H Y N I C}$ annexin A5 showed an uptake value of 4.14 $\% \mathrm{ID} / \mathrm{g}$ in EL4 lymphoma of the treated mice, ${ }^{111} \mathrm{In}$-labeled annexin A5-CCPM showed an uptake value of $8.01 \% \mathrm{ID} / \mathrm{g}$, indicating that prolonging the blood half-life of annexin A5 could lead to increased uptake of the radiotracer in apoptotic tumors. The increased tumor uptake of ${ }^{111} \mathrm{In}-$ labeled annexin A5-CCPM was not a result of increased permeability and retention effect, because ${ }^{111} \mathrm{In}$-labeled CCPM, which had longer blood half-life than annexin A5-CCPM, displayed only $2.81 \% \mathrm{ID} / \mathrm{g}$ in the treated tumors (Fig. 1B). Taken together, our data support the notion that increasing the half-life of annexin A5 improved the level of tumor uptake signal and was superior for noninvasive detection of apoptotic cells.
FIGURE 2. Control group: dual SPECT/ CT and near-infrared fluorescence optical imaging of EL4 lymphoma apoptosis with ${ }^{111}$ In-labeled annexin A5-CCPM. Mice were injected intravenously only with ${ }^{111}$ In-labeled annexin A5-CCPM. (A) Representative SPECT/CT images. (B) Representative fluorescence molecular tomographic images. (C) Representative autoradiographs of excised tumors. (D) Fluorescence images of same slides used in autoradiographic studies. ( $E$ and $F$ ) Immunohistochemical staining with caspase-3 (brown) of same slides used in autoradiographic studies. All images were acquired at $48 \mathrm{~h}$ after injection of ${ }^{111} \mathrm{In}-\mathrm{labeled}$ annexin A5-CCPM. Bar $=50 \mu \mathrm{m}$. 


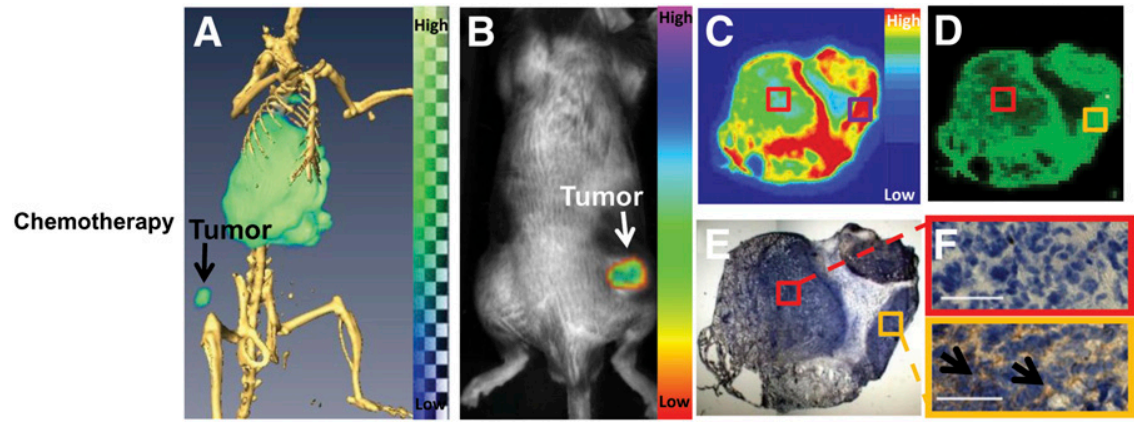

FIGURE 3. Chemotherapy group: dual SPECT/CT and near-infrared fluorescence optical imaging of EL4 lymphoma apoptosis with ${ }^{111}$ In-labeled annexin A5-CCPM. Mice in chemotherapy group received intravenous injection of ${ }^{111} \mathrm{In}$-labeled annexin A5-CCPM $24 \mathrm{~h}$ after treatment with cyclophosphamide $(25 \mathrm{mg} / \mathrm{kg})$ by intraperitoneal injection and etoposide $(19 \mathrm{mg} / \mathrm{kg})$ by intraperitoneal injection. (A) Representative SPECT/CT images. (B) Representative fluorescence molecular tomographic images. (C) Representative autoradiographs of excised tumors. (D) Fluorescence images of the same slides used in autoradiographic studies. (E and F) Immunohistochemical staining with caspase-3 (brown) of the same slides used in autoradiographic studies. All images were acquired $48 \mathrm{~h}$ after injection of ${ }^{111} \mathrm{In}$-labeled annexin A5-CCPM. Bar $=50 \mu \mathrm{m}$. Arrows in F refer to region of tumor apoptosis.

The overall strength of nanoparticle binding to target cells depends on both the affinity of the ligand-target interaction and the number of targeting ligands present on the nanoparticle surface. Multivalency effects have advantages over monovalency for binding interactions and can increase the avidity of interaction of ligands to their target receptor. This is a particularly useful feature for ligands that have a weak affinity to their target receptors in their monomer form. Nanoparticles containing multiple targeting ligands can provide multivalent binding to cell surface receptors (34). In our study, each annexin A5-CCPM nanoparticle contained approximately 40 annexin A5 molecules on the surface. Thus, the repertoire of annexin A5 was greatly expanded because of multiple, simultaneous interactions between the surface of the nanoparticle and the surface of the apoptotic cell. The in vitro studies of binding to apoptotic cells showed that ${ }^{111} \mathrm{In}$-labeled annexin A5-CCPM displayed higher detection sensitivity with radioactivity binding assay than monomeric annexin A5-fluorescein isothiocyanate with flow cytometry assay, possibly as a result of increased binding avidity (Supplemental Fig. 2).

Annexin A5 derivatives labeled with fluorescent dyes for optical imaging and with radioisotopes for nuclear imaging have been reported for detection of apoptosis in animal models and in patients, respectively $(13,14,16-25,27,28$, $31,35)$. However, it is highly desirable that an imaging probe combining a radioisotope and a near-infrared fluorescent dye be available for dual nuclear and optical imaging (36). Nuclear imaging (SPECT/CT), an established clinical imaging modality, offers excellent sensitivity and covers the whole body. However, nuclear imaging techniques are limited by relatively poor spatial resolution. Optical imaging techniques have the potential to offer real-time, highresolution images of tissues as long as they are accessible with near-infrared light. Importantly, the fluorescent signal from the imaging probes permits ex vivo analysis of excised tissues and validation of its binding to the molecular targets in vivo. The combination of 2 or more imaging techniques can therefore offer synergistic advantages over 1 modality alone in providing valuable diagnostic information. To date, several nanoparticle-based multimodal imag-

ing probes (or contrast agents) have been developed and applied for multimodality functional imaging in living animals $(27,35)$. These multimodal imaging systems have shown great promise in preclinical drug development and biomedical research. In this study, Cy-7 dye was entrapped in the core of CCPM and radioisotope chelator diethylenetriaminepentaacetic acid was conjugated on the surface of CCPM. Each micellar nanoparticle was loaded with multiple Cy7 dye molecules and ${ }^{111}$ In ions, providing a huge boost in signal intensity. As shown in both nuclear and optical imaging (Figs. 2 and 3 and Supplemental Fig. 5), ${ }^{111}$ In-labeled annexin A5-CCPM potentially can be used to locate apoptosis by whole-body nuclear and optical
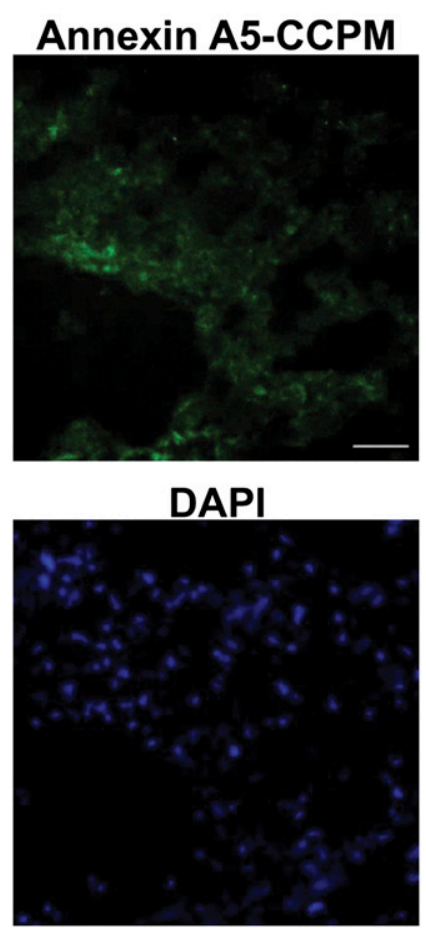

FIGURE 4. Fluorescence microscopy of EL4 lymphoma from mice treated with chemotherapy. The tumor sections were subjected to TUNEL staining (red). Signal from Cy7 loaded annexin A5-CCPM was pseudocolored green, and cell nuclei were stained with 4'-6diamidino-2-phenylindole (DAPI; blue). Bar $=50 \mu \mathrm{m}$. 
imaging. Histopathologically, ${ }^{111}$ In-labeled annexin A5CCPM revealed apoptotic areas in tumor xenografts consistent with autoradiographic and fluorescent findings of tumor sections (Figs. 2-4 and Supplemental Figs. 5 and 6).

\section{CONCLUSION}

In this study, we evaluated the potential application of ${ }^{111}$ In-labeled annexin A5-functionalized CCPMs for multimodality detection of drug-induced tumor apoptosis. In 2 tumor models, chemotherapy-induced apoptosis was readily visualized with ${ }^{111}$ In-labeled annexin A5-CCPM using both SPECT and near-infrared fluorescence imaging. Moreover, annexin A5-CCPM permitted detection of apoptotic cells at the microscopic level by optical imaging. Successful translation of ${ }^{111}$ In-labeled annexin A5-CCPM and other multimodal imaging probes into the clinic should improve the efficacy of detection of tumor apoptosis and the management of cancer patients after various therapeutic interventions.

\section{DISCLOSURE STATEMENT}

The costs of publication of this article were defrayed in part by the payment of page charges. Therefore, and solely to indicate this fact, this article is hereby marked "advertisement" in accordance with 18 USC section 1734.

\section{ACKNOWLEDGMENTS}

We thank Stephanie Deming for editing the manuscript and Qian Huang and Zhi Yang for helping with pharmacokinetic and biodistribution studies. This work was supported in part by National Institutes of Health (NIH) grants R01 CA119387 and RC2 GM092599, the John S. Dunn Foundation, the Harry S. and Isabel C. Cameron Foundation Fellowship, and the NIH Cancer Center support grant CA016672 for support of Anderson's Small Animal Imaging Facility and High-Resolution Electron Microscopy Core Facility.

\section{REFERENCES}

1. Kerr JFR, Wyllie AH, Currie AR. Apoptosis: basic biological phenomenon with wide-ranging implications in tissue kinetics. Br J Cancer. 1972;26: 239-257.

2. Tepper CG, Studzinski GP. Resistance of mitochondrial-DNA to degradation characterizes the apoptotic but not the necrotic mode of human leukemia-cell death. J Cell Biochem. 1993;52:352-361.

3. Thompson CB. Apoptosis in the pathogenesis and treatment of disease. Science. 1995;267:1456-1462.

4. Narula J, Haider N, Virmani R, et al. Apoptosis in myocytes in end-stage heart failure. N Engl J Med. 1996;335:1182-1189.

5. Fadok VA, Savill JS, Haslett C, et al. Different populations of macrophages use either the vitronectin receptor or the phosphatidylserine receptor to recognize and remove apoptotic cells. J Immunol. 1992;149:4029-4035.

6. Verhoven B, Schlegel RA, Williamson P. Mechanisms of phosphatidylserine exposure, a phagocyte recognition signal, on apoptotic t-lymphocytes. $J$ Exp Med. 1995;182:1597-1601.

7. Thiagarajan P, Tait JF. Binding of annexin-v placental anticoagulant protein i to platelets: evidence for phosphatidylserine exposure in the procoagulant response of activated platelets. J Biol Chem. 1990;265:17420-17423.
8. Koopman G, Reutelingsperger CPM, Kuijten GAM, Keehnen RMJ, Pals ST, Vanoers MHJ. Annexin-v for flow cytometric detection of phosphatidylserine expression on b-cells undergoing apoptosis. Blood. 1994;84:1415-1420.

9. Raynal P, Pollard HB. Annexins: the problem of assessing the biological role for a gene family of multifunctional calcium-binding and phospholipid-binding proteins. Biochim Biophys Acta. 1994;1197:63-93.

10. Tait JF, Gibson D. Measurement of membrane phospholipid asymmetry in normal and sickle-cell erythrocytes by means of annexin-v binding. J Lab Clin Med. 1994;123:741-748.

11. Wood BL, Gibson DF, Tait JF. Increased erythrocyte phosphatidylserine exposure in sickle cell disease: flow-cytometric measurement and clinical associations. Blood. 1996;88:1873-1880.

12. Zhang G, Gurtu V, Kain SR, Yan GC. Early detection of apoptosis using a fluorescent conjugate of annexin v. Biotechniques. 1997;23:525-531.

13. Schellenberger EA, Weissleder R, Josephson L. Optimal modification of annexin v with fluorescent dyes. ChemBioChem. 2004;5:271-274.

14. Glaser M, Collingridge DR, Aboagye EO, et al. Iodine-124 labelled annexin-v as a potential radiotracer to study apoptosis using positron emission tomography. Appl Radiat Isot. 2003;58:55-62.

15. Zijlstra S, Gunawan J, Burchert W. Synthesis and evaluation of a F-18-labelled recombinant annexin-v derivative, for identification and quantification of apoptotic cells with pet. Appl Radiat Isot. 2003;58:201-207.

16. Grierson JR, Yagle KJ, Eary JF, et al. Production of [F-18]fluoroannexin for imaging apoptosis with pet. Bioconjug Chem. 2004;15:373-379.

17. Murakami Y, Takamatsu H, Taki J, et al. F-18-labelled annexin v: a PET tracer for apoptosis imaging. Eur J Nucl Med Mol Imaging. 2004;31:469-474.

18. Blankenberg FG, Katsikis PD, Tait JF, et al. In vivo detection and imaging of phosphatidylserine expression during programmed cell death. Proc Natl Acad Sci USA. 1998;95:6349-6354.

19. Blankenberg FG, Katsikis PD, Tait JF, et al. Imaging of apoptosis (programmed cell death) with Tc-99m annexin v. J Nucl Med. 1999;40:184-191.

20. Blankenberg FG, Naumovski L, Tait JF, Post AM, Strauss HW. Imaging cyclophosphamide-induced intramedullary apoptosis in rats using Tc-99m-radiolabeled annexin v. J Nucl Med. 2001;42:309-316.

21. Blankenberg FG, Post AM, Tait JF, Blankenberg TA, Strauss HW. Apoptosis in acute and chronic abscesses [abstract]. J Nucl Med. 2001;42:333P-334P.

22. Post AM, Katsikis PD, Tait JF, Strauss HW, Blankenberg FG. Apoptosis in experimental rheumatoid arthritis [abstract]. J Nucl Med. 2001;42:128P.

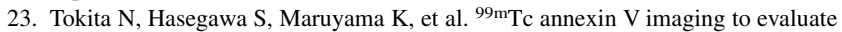
apoptosis in autoimmune myocarditis rats [abstract]. J Nucl Med. 2001;42:45P.

24. Franc BL, Song HM, Warren SM, et al. Imaging cranial suture apoptosis utilizing ${ }^{99 \mathrm{~m}} \mathrm{Tc}$ labeled annexin $\mathrm{V}$ in a rat model of suture fusion [abstract]. $\mathrm{J} \mathrm{Nucl} \mathrm{Med}$. 2002;43:346P.

25. Blankenberg FG. In vivo detection of apoptosis. J Nucl Med. 2008;49:81s-95s.

26. Schellenberger EA, Hogemann D, Josephson L, Weissleder R. Annexin v-clio: a nanoparticle for detecting apoptosis by MRI. Acad Radiol. 2002;9:S310-S311.

27. Van Tilborg GAF, Mulder WJM, Chin PTK, et al. Annexin a5-conjugated quantum dots with a paramagnetic lipidic coating for the multimodal detection of apoptotic cells. Bioconjug Chem. 2006;17:865-868.

28. Blankenberg F. To scan or not to scan, it is a question of timing: technetium$99 \mathrm{~m}$-annexin $\mathrm{v}$ radionuclide imaging assessment of treatment efficacy after one course of chemotherapy. Clin Cancer Res. 2002;8:2757-2758.

29. Yang Z, Zheng SY, Harrison WJ, et al. Long-circulating near-infrared fluorescence core-cross-linked polymeric micelles: synthesis, characterization, and dual nuclear/optical imaging. Biomacromolecules. 2007;8:3422-3428.

30. Al-Ejeh F, Darby JM, Tsopelas C, Smyth D, Manavis J, Brown MP. Apomab (r), a la-specific monoclonal antibody, detects the apoptotic tumor response to lifeprolonging and DNA-damaging chemotherapy. PLOS ONE. 2009;4:e4558.

31. Ke $\mathrm{S}$, Wen $\mathrm{X}, \mathrm{Wu} \mathrm{QP}$, et al. Imaging taxane-induced tumor apoptosis using pegylated, ${ }^{111}$ In-labeled annexin v. J Nucl Med. 2004;45:108-115.

32. Green DR, Beere HM. Apoptosis - gone but not forgotten. Nature. 2000;405: 28-29.

33. Wen X, Wu QP, Ke S, et al. Improved radiolabeling of pegylated protein: pegylated annexin $\mathrm{v}$ for noninvasive imaging of tumor apoptosis. Cancer Biother Radiopharm. 2003;18:819-827.

34. Hong S, Leroueil PR, Majoros IJ, Orr BG, Baker JR Jr, Banaszak Holl MM. The binding avidity of a nanoparticle-based multivalent targeted drug delivery platform. Chem Biol. 2007;14:107-115.

35. Schmidt V, Giacomelli C, Lecolley F, Lai-Kee-Him J, Brisson AR, Borsali R. Diblock copolymer micellar nanoparticles decorated with annexin-a5 proteins. J Am Chem Soc. 2006;128:9010-9011.

36. Li C, Wang W, Wu QP, et al. Dual optical and nuclear imaging in human melanoma xenografts using a single targeted imaging probe. Nucl Med Biol. 2006;33:349-358. 